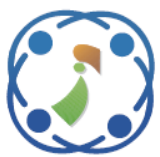

\title{
Contrast Enhancement and Visibility Restoration of Underwater Optical Images Using Fusion
}

\author{
Samarth Borker ${ }^{1 *} \quad$ Sanjiv Bonde ${ }^{1}$ \\ ${ }^{1}$ Department of Electronics and Telecommunication Engineering \\ Shri. Guru Gobind Singhji Institute of Engineering and Technology, Vishnupuri, Nanded, India \\ * Corresponding author’s Email: borkarsamarth@sggs.ac.in
}

\begin{abstract}
This paper proposes a novel method for underwater image enhancement algorithm using Principal Component Analysis (PCA) fusion. For fusion process, the dual images are derived using single underwater degraded image. The novelty of this work is the fact that it does not demand any underwater scene parameters beforehand and is independent of prior based information. Secondly, we demonstrated the successful use of homomorphic filter and adaptive histogram equalization for individual color channels followed with image smoothing as two inputs to fusion scheme. The fused image is subsequently post-processed using color constancy technique for effective results. The visual and quantitative analysis of this method is carried out with contemporary underwater dehazing and enhancement algorithms. Metrics such as entropy and quantification of restored edges is utilized to validate our findings. The outcome reveals better exposure of dark areas, enhancement of edges, increase in overall contrast and preservation of natural colors.
\end{abstract}

Keywords: Underwater visibility enhancement, Image restoration, Color correction.

\section{Introduction}

The contrast enhancement of single underwater hazy images is a challenging task for scientific exploration and computational applications. At greater depths, due to attenuation in light propagation, the underwater images are prone to inferior visibility. The artificial source of lighting is used to increase the visibility range. The presence of marine snow and small floating particle in the cone of vision of camera and image plane results in forward and backward scattering of light energy, rendering low contrast and hazy image appearance. Also, light is attenuated with a lateral distance of propagation, reducing the light energy reaching the camera, thus resulting in a loss of the natural color [1]. Thus, the primary goal of underwater image enhancement is the removal of the hazy veil and color compensation using the single underwater image.

Based on conventional haze removal algorithms, several attempts have been made to improve the underwater image visibility. The reason accounted

for the failure of conventional methods is attributed to the formation of an image scene as a multiplicative process, and hence linear enhancement is not advisable [2]. The results of traditional enhancement algorithm applied to raw underwater image is as shown in Fig. 1.

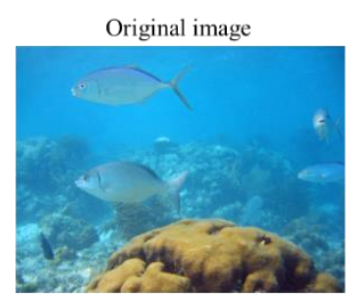

Histogram stretching

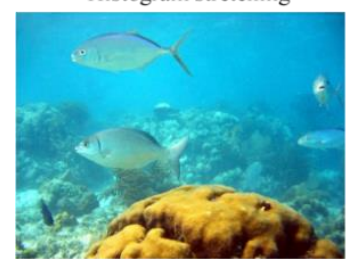

Figure 1. Conventional image enhancing methods presents drawbacks for underwater image restoration 
The major hurdle in underwater image processing is the unavailability of reference images and ground truth. As we do not have the flexibility of capturing multiple underwater images of the same scene [3], gated range lighting [4] or availability of polarization cameras to record images [5], we direct our attention to algorithm based haze removal of the single underwater hazy image. Underwater visibility enhancement and restoration are generally classified as i) Statistical prior based techniques and ii) Enhancement techniques.

In the case of prior based algorithms, as the problem is ill-poised, we presume certain statistical assumptions beforehand. The most widely adopted technique is based on such image statistical modelling is dark channel prior (DCP) [6]. It is derived from the studies and experiments that, in RGB color space, one color channel will have the least minimum intensity for underlying pixel referred to as the dark pixel. So the resulting single plane image of dark pixels constitutes a relatively dark image for the clean input image, whereas for a hazy image this dark image will constitute the image of whiteness. This image of whiteness is then quantified as haze component and is used for dehazing of natural images. This method has been widely adopted by various researchers for underwater scenario and prominent of them is found in $[7,8]$. Generally, the major limitation of such methods is, if the part of the image component resembles the whiteness of image, dehazing becomes a difficult task. The second major problem with these statistical prior based method is selection criteria of atmospheric light value and choice of filter for refinement of transmission map using edge-preserving smoothing filters.

The DCP technique has been adopted and extended for underwater image enhancement by Chiang and Chen [7]. The author separates the image into foreground and background to estimate the artificial lighting used. And then based on the object to camera distance and using energy ratios compensate the color loss. It uses predefined scattering parameters for the associated water body. But these values vary on account on structure, time and other factors, which lead to incomplete removal of haze and thus limiting its use for the generalized underwater application.

Serikawa and $\mathrm{Lu}$ [8] proposed dehazing of underwater images applying a joint trilateral filter to solve the problem of gradient reversal artifacts. Again, the authors have not addressed the problem of uneven illumination of light generated on account of artificial light used. Importantly, the authors in this work have directed the need for data fusion to address the issue of artificial light.
Galdran et al. [9] adopted and extended work of DCP by recovering the dehazed image using the red channel of RGB color space. Authors also proposed a technique to find the portion of the image affected with artificial illumination within the scene. The major issue with this algorithm is the prior assumption of red color being the most affected channel. Contrary, if we obtain the mean intensity of the underwater images for the varied set, we find that for some cases the blue color wavelength is the most attenuated channel. So this can be looked upon as a major shortcoming of this work.

$\mathrm{Li}$ et al. [10] proposed underwater image restoration method using the principle of least information loss and technique to compute global atmospheric light using optical properties. Although the results have been the best so far but fail in case of images that lack the background portion of the underwater image scene. This method is also a prior based and rely heavily on DCP and predefined residual energy ratios, which vary from case to case application.

Classical image enhancement techniques have been modified to adapt to the underwater imaging. These methods do not depend on physical modeling of underwater scenario. The most popular method is underwater image and video enhancement using fusion to combine different weighted images using saliency, luminance, and chrominance via filtering [2]. This was the first recorded work for the enhancement of underwater images using fusion approach based on Laplacian pyramid. The authors also validated the selection of white balancing algorithm for underwater images. Although the contrast of the output images appears increased, the problem associated with it is, as reflected in the results section, the processed images are not uniformly enhanced and does not appear natural.

Inherently very less research has been carried out in underwater image enhancement using PCA fusion. As to our understanding, the only available work in underwater image processing using PCA fusion is proposed by [11]. The authors of this work have taken the same reference image as two inputs to the fusion approach, without giving attention to the underwater propagation of light and formation of the image together with the generic problem of underwater attenuation and scattering issues.

In our proposed work, using a novel approach we address the above shortcomings like a loss in visibility due to color attenuation, image contrast reduction on account of scattering and rectify the color artifact issue based on Shades of Gray algorithm [12]. In this paper, we derive the two inputs to the fusion using a single underwater degraded 
image. The two inputs are derived using a homomorphic filter and adaptive histogram equalization for individual color channels followed with image smoothing. The fused image is subsequently post-processed using color constancy technique for effective results. The main contribution of the paper are summarized as follows:

* The proposed method does not demand any underwater scene parameters beforehand and is independent of prior based information.

* We are the first to demonstrate the successful use of Homomorphic filter and adaptive histogram equalization for individual color channels followed with image smoothing as two inputs to fusion scheme.

* A robust PCA based fusion followed with color constancy algorithm serves the purpose of image dehazing and yield effective results as compared to conventional methods of image enhancement and restoration for underwater applications.

The contents of this paper are organized as follows: In Section 2, selection criteria of two inputs for the fusion process are highlighted. Section 3 presents the PCA based fusion algorithm. Section 4 describes the visual and quantitative analysis and the discussion on the choice of selection of metrics used to gauge the enhancement criteria and finally, we draw a conclusion and direct future work in Section 5 .

\section{Proposed Method for Underwater Image Enhancement}

In this paper, we introduce enhancement algorithm based on principal component analysis fusion method. The simplified block representation of the proposed underwater image enhancement is as shown in Fig. 2.
The selection of suitable inputs to fusion forms the crucial component of the proposed algorithm. Improper selection of input methodologies results in the ineffective enhancement and unrealistic restoration. In the following sections, we have explained the reasons for selecting the choice of Homomorphic filtering and Adaptive histogram equalization as two inputs derived from the raw underwater hazy image.

\subsection{Homomorphic filtering}

To overcome the problem of diminished underwater visibility, an artificial lighting source is used. This sets up a non-uniform illumination pattern. We propose to use homomorphic filtering to tackle the issue of non-uniform illumination. The image $f(x, y)$ has two components, illumination component $i(x, y)$ and reflection component $r(x, y)$ expressed as:

$$
f(x, y)=i(x, y) \cdot r(x, y)
$$

The illumination component features slow spatial variations, whereas the reflectance tends to change at a higher rate. So we can associate low frequencies with illumination and high frequencies of the Fourier transform with reflectance respectively. So by applying a homomorphic filter, we filter out nonillumination component and restore the image by suppressing the low-frequency components and amplifying high-frequency components. Following steps summarize the implementation of homomorphic filtering [13]:

Step 1: Apply natural logarithm to isolate $i(x, y)$ and $r(x, y)$.

$$
f(x, y)=\ln i(x, y)+\ln r(x, y)
$$

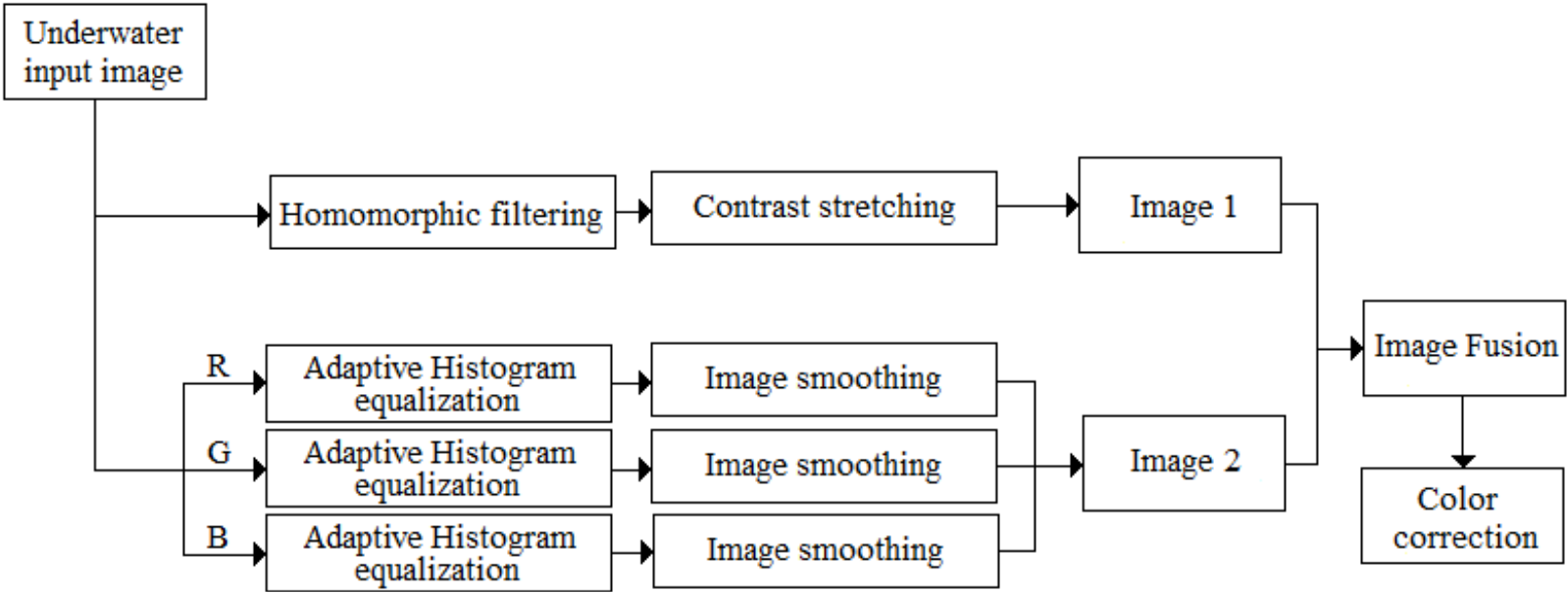

Figure 2. Block representation of the proposed underwater image enhancement 
Step 2: Using Fourier transform, map the image to the frequency domain.

$$
\begin{aligned}
& \mathfrak{I}\{f(x, y)\}=\mathfrak{I}\{\ln i(x, y)\}+\mathfrak{I}\{\ln r(x, y)\} \\
& f(u, v)=F_{i}(u, v)+F_{r}(u, v)
\end{aligned}
$$

where $F_{i}(u, v)$ and $F_{r}(u, v)$ are the respective Fourier transform of $\ln i(x, y)$ and $\ln r(x, y)$.

Step 3: In our approach, we first design a low pass Gaussian filter and then obtain high pass filter equivalent from it. The Gaussian filter function is given as:

$$
G(x, y)=\frac{1}{2 \pi \sigma^{2}} e^{-\left(\frac{x^{2}+y^{2}}{2 \sigma^{2}}\right)}
$$

where $\sigma$ is the standard deviation of the distribution and $(x, y)$ is the pixel coordinate position. The Gaussian low pass filter has the property of retaining sharp edges and detail information as compared to other low pass filters.

Step 4: Using step 3, we obtain filter function $H(u, v)$ in frequency domain to compute filtered component $S(u, v)$ as:

$$
\begin{aligned}
& S(u, v)=H(u, v) F(u, v) \\
& S(u, v)=H(u, v) F_{i}(u, v)+H(u, v) F_{r}(u, v)
\end{aligned}
$$

Step 5: Using inverse Fourier transform, map the filter function back to the spatial domain.

$\mathfrak{I}^{-1}\{S(u, v)\}=\mathfrak{I}^{-1}\left\{H(u, v) F_{i}(u, v)+H(u, v) F_{r}(u, v)\right\}$

Step 6: Homomorphic filtered image is then obtained from below equation as:

$$
J(x, y)=\exp \{s(x, y)\}
$$

\subsection{Contrast stretching}

The image obtained $J(x, y)$ needs to be further processed to increase the dynamic contrast range. The advantage of converting the image to YUV color space is that its brightness image $Y$ and chroma images (color component) $U$ and $V$ are distinct and holds advantage of operating directly on $Y$ component. We transform the RGB to YUV color space using the following set of equations:

$$
\left[\begin{array}{l}
Y \\
U \\
V
\end{array}\right]=\left[\begin{array}{lll}
0.299 & 0.557 & 0.144 \\
-.147 & -.288 & 0.436 \\
0.615 & -.514 & -.100
\end{array}\right]\left[\begin{array}{l}
R \\
G \\
B
\end{array}\right]
$$

Next, we apply contrast adjustment algorithm to only $Y$ channel (Luminance channel) to increase the dynamic range of an image without affecting the Chroma components. The contrast adjusted image is expressed as [14]:

$$
\begin{aligned}
Y^{\prime}= & \left(\frac{\left(Y-\text { low }_{\text {in }}\right)}{\left(\text { high }_{\text {in }}-\text { low }_{\text {in }}\right)}\right)\left(\left(\text { high }_{\text {out }}-\text { low }_{\text {out }}\right)\right) \\
& + \text { low }_{\text {out }}
\end{aligned}
$$

where $Y$ represent input image pixel under consideration high $_{\text {in }}$, low $_{\text {in }}$, high out $_{\text {, }}$ low $_{\text {out }}$ corresponds to input image high intensity, input image low intensity, output image high intensity and output image low intensity respectively.

This operation performs scaling of the original data linearly thereby not losing any information other than the intensity of original pixels. We have stretched the values for the lower limit and the upper limit to $1 \%$ of lower and higher pixel intensities respectively. Then we transform the YUV space color image back to three channel RGB color space using the following set of equations:

$$
\left[\begin{array}{l}
R^{\prime} \\
G^{\prime} \\
B^{\prime}
\end{array}\right]=\left[\begin{array}{lll}
1.000 & 0.000 & 1.140 \\
1.000 & -.395 & -.581 \\
1.000 & 2.032 & 0.000
\end{array}\right]\left[\begin{array}{l}
Y^{\prime} \\
U \\
V
\end{array}\right]
$$

The reason behind selecting the YUV color space for this operation is that it is very close to human vision imaging science. We also tested it with $\mathrm{YCbCr}$ color space [15], but the result was visually not appealing.

\subsection{Adaptive histogram equalization}

The second input to PCA fusion scheme is derived from adaptive histogram equalization. Histogram equalization is one of the major technique of image enhancement application. Since it stretches the contrast value globally, it leads to the information loss, especially in dark regions. To address this problem, adaptive histogram equalization was incorporated. It works at predefined blocks also called as windowing, then the mapping of gray level is carried out for window center thereby improving the edge definitions in each region of an image. This 
technique on account of its inherent structure results in over enhancement at some pixels and also introduces image noise. To overcome this problem, we have used a median filter to smoothen the image.

As it is pointed out in Section 1 and upon experimentation, we found that certain color channel has the least mean intensity as compared to other channels. So there arises a need for individual pixel distribution for gray levels. So we separated the three channels and individually applied localized histogram equalization. To avoid the oversaturation of pixels and to isolate out of range noise from legitimate image edge details, we apply a smoothening filter to image $x(i, j)$ given as:

$$
y[m, n]=\operatorname{median}\{x(i, j),(i, j) \in w\}
$$

where $w$ represents a neighborhood centered around $[m, n]$ defined by the user. Compared to $2 \times 2$ averaging filter, median filter preserves edge and contour details, at the same time sharp peripheral noise is eliminated.

\section{Proposed PCA based Fusion Algorithm}

PCA is a mathematics based tool that transforms correlated variable to uncorrelated variables defined as principal components. The following steps are used to project two column vector to $2 \mathrm{D}$ subspaces $[16,17]$ :

Step 1: The data is arranged in column vectors, generating a matrix $Z$ of $2 \mathrm{xN}$ size.

Step 2: Empirical mean vector $M_{e}$ of size $1 \times 2$ is computed.

Step 3: Empirical mean vector $M_{e}$ is subtracted from matrix obtained in step 1 . This results in matrix $X$ of $2 \mathrm{xN}$ dimension.

Step 4: Covariance matrix $C$ is obtained using expression $C=X X_{T}$.

Step 5: Eigenvector $V$ and eigenvalue $D$ is computed for covariance matrix $C$.

Step 6: Eigenvector $V$ and eigenvalue $D$ are then arranged in descending eigenvalue. The dimension of $V$ and $D$ is $2 \times 2$.

Step 7: Using primary column of $V$, corresponding to the larger eigenvalue, obtain $A_{l}$ and $A_{2}$.

$$
A_{1}=\frac{V(1)}{\sum V} \text { and } A_{2}=\frac{V(2)}{\sum V}
$$

Step 8: The fused image $I_{f}(x, y)$ is then obtained using the following expression:

$$
I_{f}(x, y)=A_{1} I_{1}(x, y)+A_{2} I_{2}(x, y)
$$

where $I_{1}(x, y)$ and $I_{2}(x, y)$ are the two input image to be fused.
Compared to transform based fusion algorithms like discrete wavelet transform (DWT), the proposed pixel level principal component analysis (PCA) is simple and does not introduce any artifacts. The DWT based fusion methods have a tendency to introduce additive noise in the resultant image [18].

\subsection{Post-processing using color constancy}

So far we have addressed the problem of nonuniform illumination and color attenuation on account of the artificial source of lighting and wavelength dependency of underwater transmission in the image formation process respectively. There is yet another problem of scattering on account of suspended floating particles between camera and object scene, which results in color offsets or unwanted color cast. We propose using the color constancy algorithm to eliminate this problem [12]. We experimented with white patch algorithm, Grey Edge algorithm and Shades of Gray algorithm [19]. We obtained satisfactory results with Shades of Gray color constancy algorithm. The probable reason for the failure of white patch algorithm is attributed to partial specular reflection and gray edge algorithm needs high contrast and detailed edge information for optimized performance [2].

Shades of Gray algorithm was presented by Finlayson and Trezzi, based on the assumption that scene average is a function of the shade of gray. It computes the weighted mean of the pixel intensity by allotting higher weight to the pixel with high intensity using Minkowski - norm $p$, given as:

$$
\left(\frac{\int(f(x))^{p} d x}{\int d x}\right)^{1 / p}=k e
$$

where $f(x)$ is the input image, $(x)$ is the pixel coordinate and $k$ is constant. Basically, Shades of Grey is a trade-off between the Gray world $(p=1)$ and MaxRGB algorithm $(p=\infty)$.

\section{Experimental Results and Discussion}

The performance metrics play an essential role in the assessment of the degree of restoration. In the case of underwater image enhancement field, this becomes difficult by virtue of the absence of reference images or unavailability of ground truth. So as to evaluate the performance of the proposed algorithm we have used the similar set of images used by earlier researchers working in this field and same assessment metric so as to compare the work on the common platform. The contemporary works that we 
have used for comparison include work of Ancuti et al., [2], Chiang and Chen [7], Serikawa and Lu [8], Galdran et al., [9], and Li et al., [10].

\subsection{Qualitative assessment}

Using qualitative (visual) comparison as shown in Fig. 3, we have compared our works with state-ofthe-art techniques as mentioned above.

Galdran et al., [9] has set up an online repository and provided a platform for comparison and quantification of visual enhancement of underwater images, which have helped us comparing our work with contemporary techniques.

As shown in Fig. 3, the images obtained by applying our algorithm is characterized by enhanced contrast, optimized visibility and retaining a natural appearance. The algorithm of Ancuti et al increases the contrast but over enhances the image, rendering it an unnatural look. Also, some portion of the image still remains dark. Chiang and Chen's algorithm fails to unveil the details of the hazy portion of an image.

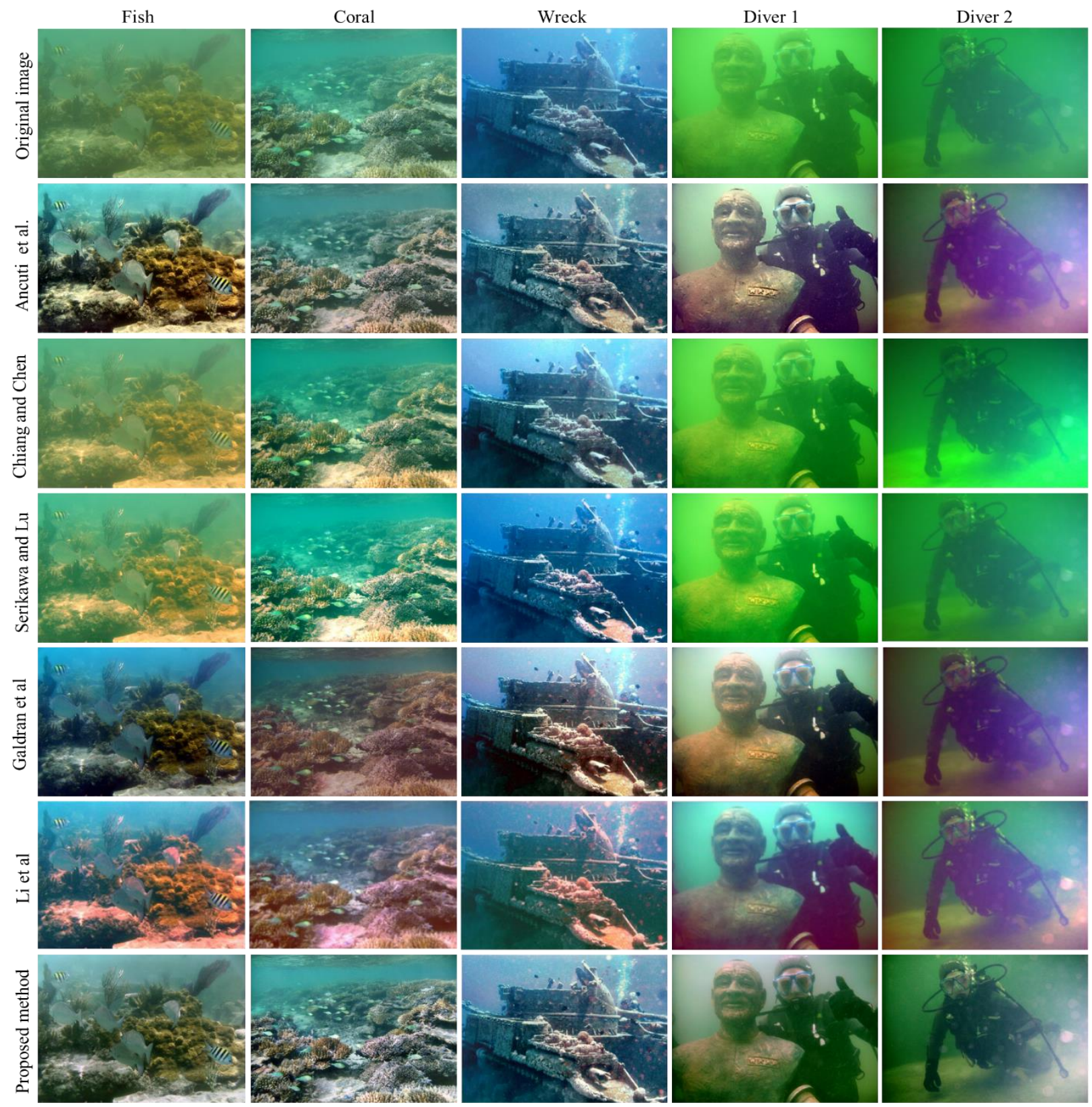

Figure 3. From top to bottom; Row 1: Input underwater images 512x384, Row 2: Ancuti et al. (2012) results, Row 3: Chiang and Chen (2012) results, Row 4: Serikawa and Lu results (2014), Row 5: Galdran et al. (2015) results, Row 6: Li et al. (2016) results and Row 7: Our results. 
Serikawa and $\mathrm{Lu}$ are able to increase the visual aspect of the raw image but fail completely for the images when one color channel dominates the other two. This has been a major problem with DCP based methods as also observed with Chiang and Chen. Galdran et al are able to restore the clarity of the dehazed image, but the unwanted color cast is observed in specific raw images. Li et al., do increase the brightness and details of the raw image, still, the problem of color cast is prevalent. As far as our results are concerned, one can observe that we are able to enhance contrast, improve edge details together with natural visibility. The problem of the unwanted color cast is totally addressed. Also compared to existing methods as mentioned above, we are able to expose the shadowy regions much effectively.

We have also compared the visual results of our algorithm with standard DCP based algorithms. As shown in Fig. 4, the standalone DCP method is not sufficient to enhance the visibility or able to retain the natural color of raw underwater images and hence demands suitable modification or extension of the existing work. We have compared our result with $\mathrm{He}$ et al., [6], Meng et al., [20] and Zhu et al., [21]. The proposed method is able to enhance edge details and preserve the depth of view. Also, it is observed that DCP methods generally fail to restore visibility at depth or background area of the image scene.

\subsection{Quantitative Assessment}

We have followed the preceding works for the choice of quantitative assessment metrics [10]. We have used entropy [22] and the ratio of visible edges metric [23] to compare and validate our results. These two metrics are extensively utilized to appraise the performance of various underwater restoration algorithms.

The entropy metric quantifies the increased contrast amount and outlines detail information in dehazed images. Poor images characterize lower entropy and vice versa.
Table 1. Average values of Entropy $(\mathrm{H})$ and Visible edge ratio (VER).

\begin{tabular}{|l|l|c|c|}
\hline Image & \multicolumn{1}{|c|}{ Method } & H & VER \\
\hline \multirow{3}{*}{ Fig. 3 } & Ancuti et al. [2] & 7.77 & 3.4281 \\
\cline { 2 - 4 }$\{$ Fish, & Chiang and Chen [7] & 6.85 & -0.4863 \\
\cline { 2 - 4 } Coral, & Serikawa and Lu [8] & 6.74 & 0.0372 \\
\cline { 2 - 4 } Wreck, & Galdran et al. [9] & 7.75 & 2.1521 \\
\cline { 2 - 4 } Diver1 & Li et al. [10] & 7.81 & 3.4721 \\
\cline { 2 - 4 } Diver 2\} & Proposed method & 7.61 & 3.7777 \\
\hline
\end{tabular}

Table 1 shows that our algorithm exhibits comparatively higher value of entropy $(\mathrm{H})$ as to that of recent methods. Whereas the parameter, visible edge ratio (VER) computes the ratio of edges, invisible in raw images to those visible in dehazed images. A higher value of VER is the characteristic feature of enhanced visibility. Table 1 validates that our algorithm produces the better results compared to contemporary methods.

\section{Conclusion}

In this work, we have presented the enhancement of single underwater images using PCA based fusion rule. Two inputs for the fusion scheme are derived from homomorphic filter and adaptive histogram equalization methods. The inputs to fusion address the problem associated with underwater imaging such as non-uniform illumination and low visibility due to color attenuation. The fused image is subsequently post-processed using color constancy algorithm. The proposed algorithm is tested for standard underwater images as used by contemporary researchers working in this domain. We obtained the highest value of metric for visible edge ratio, and for entropy, we managed to obtain a value comparable to that of contemporary techniques. As far as visual comparison is concerned, we obtained the best possible enhancement even in dark regions, where existing methods fail. We exposed the ineffectiveness of standard enhancement techniques like histogram equalization, histogram stretching for underwater image restoration.

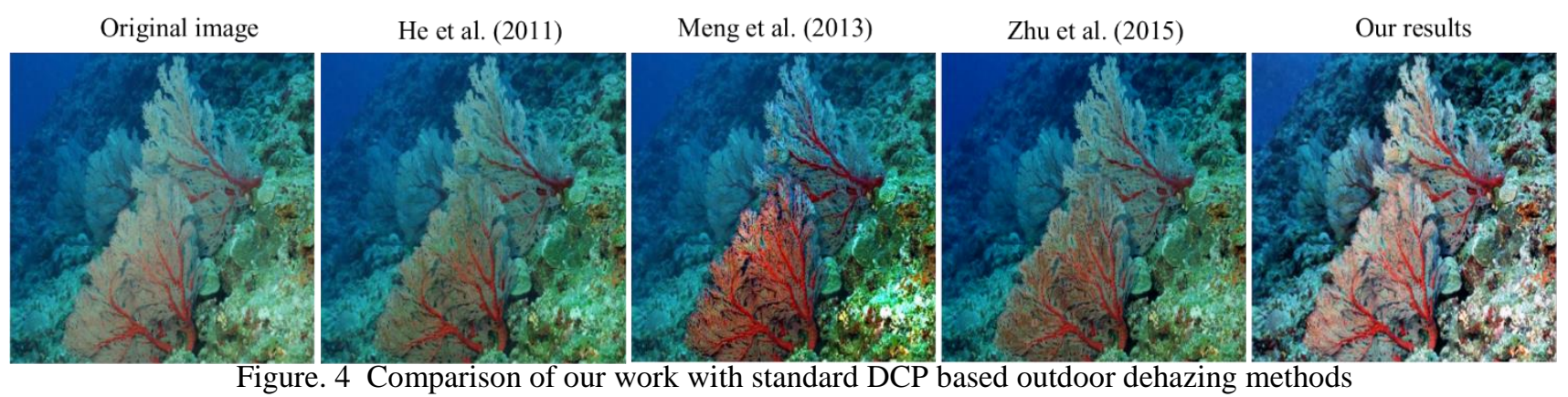


We have further demonstrated that underwater image restoration using DCP is not effective for the fact that the image formation in the outdoor and underwater scenario is different. The outcomes of qualitative and quantitative analysis validate our claims for enhanced visibility of edges and increased entropy values. The visual outcomes also reveal efficient restoration of images with preservation of natural color. In future work, we shall undertake underwater image mosaicking and video processing.

\section{References}

[1] R. Schettini and S. Corchs, "Underwater Image Processing: State of the Art of Restoration and Image Enhancement Methods", EURASIP Journal on Advances in Signal Processing, Article ID 746052, pp.1-14, 2010.

[2] C. Ancuti, C. O. Ancuti, T. Haber, and P. Bekaert, "Enhancing Underwater Images and Videos by Fusion", In: Proc. of IEEE Conf. on Computer Vision and Pattern Recognition, Providence, RI, pp.81-88, 2010.

[3] S. G. Narasimhan and S. K. Nayar, "Contrast Restoration of Weather Degraded Images", IEEE Transactions on Pattern Analysis and Machine Intelligence, Vol.25, No.6, pp.713-724, 2003.

[4] D. M. He and G. L. Seet, "Divergent Beam Lidar Imaging in Turbid Water", Optics and Lasers in Engineering, 2004.

[5] Y. Y. Schechner and Y. Averbuch, "Regularized Image Recovery in Scattering Media", IEEE Transactions on Pattern Analysis and Machine Intelligence, Vol.29, No.9, pp.1655-1660, 2007.

[6] K. He, J. Sun and X. Tang, "Single Image Haze Removal using Dark Channel Prior", IEEE Transactions on Pattern Analysis and Machine Intelligence, Vol.33, No.12, pp.2341-2353, 2011.

[7] J. Y. Chiang and Y. C. Chen, "Underwater Image Enhancement by Wavelength Compensation and Dehazing", IEEE Transactions on Image Processing, Vol.21, No.4, pp.1756-1769, 2012.

[8] S. Serikawa and H. Lu, "Underwater Image Dehazing using Joint Trilateral Filter", Elsevier Computers and Electrical Engineering, Vol.40, No. 1, pp.41-50, 2014.

[9] A. Galdran, D. Pardo, A. Picón, and A. AlvarezGila, "Automatic Red-Channel Underwater Image Restoration", Journal of Visual Communication and Image Representation, Vol.26, pp.132-145, 2015.

[10] C. Li, J. Guo, B. Wang, R. Cong, Y. Zhang, and J. Wang, "Single Underwater Image Enhancement based on Color Cast Removal and
Visibility Restoration", Journal of Electronic Imaging, Vol.25, No.3, pp.330121-15, 2016.

[11] M. K. Ansar and V. Krishnan, "Performance Evaluation of Image Fusion Algorithms for Underwater Images-A study based on PCA and DWT", International Journal of Image, Graphics and Signal Processing, Vol.12, pp.6569, 2014.

[12] J. Van de Weijer, T. Gevers, and A. Gijsenij, "Edge based Color Constancy", IEEE Transactions on Image Processing, Vol.16, No.9, pp.2207-2214, 2007.

[13] S. A. Saleh and H. Ibrahim, "Mathematical Equations for Homomorphic Filtering in Frequency Domain: A Literature Survey", In: Proc. of International Conf. on Information and Knowledge Management, Singapore, Vol.45, 2012.

[14] J. Banerjee, R. Ray, S. R. K. Vadali, S. N. Shome, and S. Nandy, "Real Time Underwater Image Enhancement: An Improved Approach for Imaging with AUV-150," Sadhana, Vol.41, No.2, pp.225-238, 2016.

[15] B. Wang, X. Chang, and C. Liu, "Skin Detection and Segmentation of Human Face in Color Images", International Journal of Intelligent Engineering and Systems, Vol.4, No.1, pp.10-17, 2011.

[16] V.P.S. Naidu and J.R. Raol, "Pixel-level Image Fusion using Wavelets and Principal Component Analysis", Defence Science Journal, Vol.58, No.3, pp. 338-352, 2008.

[17] D. M. Rafi and C.R. Bharathi, "Optimal Fuzzy Min-Max Neural Network (FMMNN) for Medical Data Classification Using Modified Group Search Optimizer Algorithm", International Journal of Intelligent Engineering and Systems, Vol.9, No.3, pp.1-10, 2016.

[18] R. S. Agarwal, S. Rajkumar, K. Marimuthu, and G. Rajasekaran, "CT and MRI Image Fusion Based on Discrete Wavelet Transform and Type-2 Fuzzy Logic", International Journal of Intelligent Engineering and Systems, Vol.10, No.3, pp. 355-362, 2017.

[19] G. D. Finlayson and E. Trezzi, "Shades of Gray and Color Constancy", In: Twelfth Color Imaging Conference: Color Science and Engineering Systems, Technologies, and Applications, Scottsdale, Arizona, USA, pp.3741, 2004.

[20] G. Meng, Y. Wang, J. Duan, S. Xiang, and C. Pan, "Efficient Image Dehazing with Boundary Constraint and Contextual Regularization," In: Proc. of IEEE International Conference on 
Computer Vision, Vol.25, No.6, pp.617-624, 2013.

[21] Q. Zhu, J. Mai, and L. Shao, "A Fast Single Image Haze Removal Algorithm using Color Attenuation Prior", IEEE Transactions on Image Processing, Vol.24, No.12, pp.35223533, 2015.

[22] Y. Yang, D. S. Park, S. Huang, and N. Rao, "Medical Image Fusion via an Effective Wavelet based Approach", EURASIP Journal on Advances in Signal Processing, Article Id: 579341, pp.1-10, 2010.

[23] N. Hautière, J. P. Tarel, D. Aubert, and É. Dumont "Blind Contrast Enhancement Assessment by Gradient Ratioing at Visible Edges", Image Analysis and Stereology, Vol.27, No.2, pp.87-95, 2008. 